\title{
Vivencias de madres mapuches durante la hospitalización de sus hijos, en un hospital de alta complejidad del sur de Chile
}

\section{Mapuche mothers 'lives during the hospitalization of their children, in a high complexity hospital in southern Chile}

\section{Experiências de mães mapuche durante a hospitalização de seus filhos, em um hospital de alta complexidade no sul do Chile}

\author{
Edith Rivas Riveros ${ }^{1}$, ORCID 0000-002-9832-4534 \\ Verónica García Silva², ORCID 0000-0001-9335-6724 \\ Yaqueline Catalán Melinao ${ }^{3}$, ORCID 0000-0001-6179-0323 \\ ${ }^{13}$ Departamento de Enfermería, Facultad de Medicina. Universidad de La Frontera. \\ Temuco, Chile \\ ${ }^{2}$ Hospital Hernán Henríquez Aravena. Temuco, Chile
}

Recibido: 09/03/2019

Aceptado: 03/12/2019

\begin{abstract}
Resumen: Introducción: Cuando el cuidado cultural de enfermería reconoce el valor de la cultura, las creencias y modo de vida de los individuos, este se vuelve integral. La pregunta fue ¿Cuál es la vivencia de madres mapuches durante la hospitalización de sus hijos en un hospital de alta complejidad del sur de Chile, 2017-2018? Objetivo general: Develar la vivencia de madres mapuches durante la hospitalización de sus hijos en un hospital de alta complejidad del sur de Chile. Metodología: Se trabajó con etnografía dirigida, con una muestra intencionada de 9 participantes, los criterios de inclusión fueron: madres mapuches hablantes de mapudungun con hijos hospitalizados en servicios pediátricos, en hospital de alta complejidad; que accedan a dar una entrevista grabada, previa firma de consentimiento informado. Se recolectan los datos mediante observación participante, entrevistas en profundidad y notas de campo. Resultados: En la metacategoría vivencia de madres mapuches se identifican las subcategorías: sentido pertenencia a su cultura; complementariedad en la atención, calidad en la atención y discriminación. Conclusión: Se aprecia contrariedades, decepciones y complicaciones en legitimar la diversidad cultural y social, que se traducen en impedimentos, para el éxito de las estrategias de coherencia cultural en salud, ya que emergen trazos de discriminación. Se manifiesta integralidad del mundo indígena que se expresa en las personas a través de sus valores propios de la cultura.
\end{abstract}

Palabras claves: enfermería transcultural; población indígena; madres; hospitalización

Abstract: Introduction: When cultural nursing care recognizes the value of the culture, beliefs and way of life of individuals, it becomes integral. The question was: What is the experience of Mapuche mothers 
during the hospitalization of their children in a high complexity hospital in southern Chile, 2017-2018? Course objective: To reveal the experience of Mapuche mothers during the hospitalization of their children in a high complexity hospital in southern Chile, 2017-2018. Methodology: The study was conducted on the targeted ethnography with an intentional sample of 9 participants. The inclusion criteria were: Mapuche mothers who speak Mapudungun with children hospitalized in pediatric services, in a high complexity hospital, who agreed to give a recorded interview after signing an informed consent. Data were collected through participant observation, in-depth interviews and field notes. Results: In the metacategory experience of Mapuche mothers, the subcategories were sense of belonging to their culture, complementarity of care, quality of care and discrimination. Conclusion: There are contradictions, disappointments and complications in legitimizing cultural and social diversity, which translate into obstacles to the success of strategies for cultural coherence in health, as traces of discrimination emerge. The comprehensive nature of the indigenous world that expresses itself in people through their own values of culture is manifested.

Keywords: transcultural nursing; indigenous population; mothers; hospitalization

Resumo: Introdução: Quando o cuidado cultural de enfermagem reconhece o valor da cultura, crenças e modo de vida dos indivíduos, torna-se integral. A pergunta foi: Qual a experiência das mães mapuches durante a internação de seus filhos em um hospital de alta complexidade no sul do Chile, 2017-2018? Objetivo do curso: Revelar a experiência de mães mapuches durante a hospitalização de seus filhos em um hospital de alta complexidade no sul do Chile, 2017-2018. Metodologia: Foi realizado trabalho com etnografia direcionada, com uma amostra intencional de 9 participantes, os critérios de inclusão foram: mães mapuches que falam Mapudungun com crianças internadas em serviços pediátricos, em um hospital de alta complexidade; que concordam em dar uma entrevista gravada, após assinarem um termo de consentimento livre e esclarecido. Os dados são coletados por meio de observação participante, entrevistas em profundidade e notas de campo. Resultados: Na experiência de metacategoria de mães mapuches, identificam-se as subcategorias: sentimento de pertencimento à sua cultura; complementaridade do cuidado, qualidade do atendimento e discriminação. Conclusão: Existem contradições, desapontamentos e complicações na legitimação da diversidade cultural e social, que se traduzem em empecilhos, para o sucesso de estratégias de coerência cultural em saúde, à medida que surgem traços de discriminação. A integralidade do mundo indígena que se expressa nas pessoas através de seus próprios valores de cultura se manifesta.

Palavras-chave: enfermagem transcultural; população indígena; mães; hospitalização

Correspondencia: Edith Rivas Riveros, e-mail: edith.rivas@ufrontera.cl 


\section{Introducción}

La disciplina de enfermería se caracteriza por su formación ética, compromiso social y carácter holístico, asimismo tiene el deber de generar conocimientos en el área de la salud intercultural para contribuir al desarrollo y fortalecimiento del quehacer en concordancia con las estrategias ministeriales de salud (Minsal) de Chile, el cual expone como prioridad aportar a la disminución de las brechas de inequidad en salud indígena a través de la construcción participativa de estrategias de salud a nivel de servicios de salud y secretarías regionales ministeriales (Seremi) (1). De esta forma se reconoce la diversidad, se promueve la complementariedad entre sistemas médicos y se provee servicios de salud adecuados que respondan a las necesidades, derechos y perfiles epidemiológicos específicos de esta población (1-3).

En la actualidad en Chile, el Programa Especial de Salud y Pueblos Indígenas opera en los servicios de salud de casi todo el país con énfasis en los lugares de mayor concentración de población indígena. La participación de los pueblos indígenas en el diseño, implementación y evaluación ha sido una estrategia clave y se agrupa en tres componentes: (a) equidad, (b) interculturalidad, y (c) participación. La equidad busca contribuir a disminuir las brechas en el acceso a la atención de salud oportuna y de calidad, a través de mejoras en la accesibilidad, calidad y pertinencia cultural de la prestación de servicios a través de facilitadores interculturales, asesores culturales en la red de servicios, oficinas interculturales y de señal intercultural, habilitadas en los establecimientos de salud, para orientar a los usuarios indígenas. El enfoque de salud intercultural incentiva la formación y desarrollo de equipos de salud capaces de respetar, comprender y responder apropiadamente a las propuestas y necesidades de las comunidades y organizaciones indígenas ligadas al tema de la salud. El tercer componente del programa busca favorecer la participación de los pueblos indígenas en la planificación, implementación y evaluación de estrategias destinadas a mejorar su estado de salud (4-5). La presente investigación tiene como referente teórico la teoría de la diversidad y universalidad de los cuidados culturales propuesta de Madeleine Leininger (6).

La significancia social del estudio se relaciona con el objetivo estratégico de las inequidades en pueblos indígenas (3), por ello es relevante y necesario para las enfermeras (os) conocer la vivencia de los pueblos indígenas, considerando las políticas implementadas, por lo cual se propone una investigación cualitativa con enfoque etnográfico, con la pregunta: ¿cuál es la vivencia de madres mapuches durante la hospitalización de sus hijos en servicios pediátricos en un hospital del sur de Chile?, con el propósito de contribuir al conocimiento enfermero en el ámbito de la Gestión del Cuidado Intercultural.

\section{Objetivo general}

Develar vivencias de madres mapuches durante la hospitalización de sus hijos en un hospital de alta complejidad del sur de Chile, año-2018.

\section{Material y método}

Se realiza una investigación cualitativa que trabaja una etnografía enfocada ya que permite la aproximación a un fenómeno tal como lo experimenta un grupo, en un contexto determinado, examinando experiencias dentro de una cultura en entornos particulares (7-9). Este método permite 
el abordaje desde la perspectiva de los participantes, o desde la visión émica, pero en un sentido muy específico, con respecto a ciertas situaciones, actividades y acciones (10).

A diferencia de la etnografía clásica basada en la experiencia, la etnografía enfocada es de corto alcance y no continua, por lo que puede ser utilizada para investigar fenómenos que impliquen visitas de campo a corto plazo, como sucede con los pacientes hospitalizados. Caracterizándose por el alto nivel de análisis requerido, utilización de tecnologías, notas, transcripciones y realización de codificación y análisis secuencial (10).

Los criterios de inclusión propuestos para esta investigación fueron: madres mapuches hablantes de mapudungun (lengua mapuche) con hijos hospitalizados en servicios pediátricos en un hospital de alta complejidad, que accedan a dar una entrevista grabada, previa firma de formulario de consentimiento informado. El muestreo fue intencional, teniendo en cuenta los casos disponibles de acuerdo a los criterios de inclusión, bajo esta perspectiva se contó con 9 participantes. La recolección de los datos se realizó por medio de observación participante, entrevistas en profundidad realizadas a las informantes durante el periodo de hospitalización de sus hijos y notas de campo surgidas de la observación y entrevistas. Respecto a las entrevistas, estas fueron grabadas y transcritas de manera textual por los propios investigadores. La información recolectada a través de los relatos se fue analizando de forma inmediata, hasta alcanzar la saturación de la información, es decir, hasta no obtener datos nuevos y en el análisis de estos, la redundancia fue evidente. A partir de la información suministrada se determinaron los códigos y las categorías de análisis, seleccionando los textos significativos, en base a criterio temático, en busca de expresiones referentes a la vivencia de las madres durante la hospitalización de sus hijos.

Se trabajó triangulación por investigador, de forma que el investigador principal y coinvestigadores realizan análisis de forma independiente, posteriormente se comparte encontrándose algunas diferencias, por lo que se realiza relectura y un nuevo análisis, para finalmente lograr concordancia. De esta manera, emerge una metacategoría y las cuatro categorías intermedias.

Respecto a los criterios de rigor, se trabaja: dependencia, credibilidad, auditabilidad y transferibilidad de Guba y Lincoln (11). Se respetaron los principios éticos en investigación que involucra seres humanos de Ezekiel Emanuel (12).

\section{Resultados}

Los resultados se obtuvieron a partir de la recolección de la información por medio de observación participante, notas de campo y entrevistas en profundidad realizadas a 9 informantes claves que cumplieron con los criterios de inclusión y que aceptaron voluntariamente participar en el estudio previa firma del consentimiento informado. Se llevaron a cabo 18 entrevistas con un tiempo promedio de 60 minutos cada una.

Todas las participantes vivían en la región de la Araucanía de Chile, con edades comprendidas entre 32 y 36 años, 5 de ellas con escolaridad básica incompleta, 3 con educación media completa y 1 con educación superior completa.

Tras el análisis de la información surgió la metacategoría: vivencia de madres mapuches y se identificaron las subcategorías: sentido pertenencia a su cultura, complementariedad en la atención, calidad en la atención y discriminación. 
Tabla 1: Resumen de metacategorías, subcategorías y unidades de análisis

\begin{tabular}{|c|c|c|}
\hline Metacategorías & Subcategorías & Unidades de análisis \\
\hline \multirow{4}{*}{$\begin{array}{l}\text { Vivencia de } \\
\text { madres mapuches }\end{array}$} & $\begin{array}{ll}\text { Sentido } & \mathrm{de} \\
\text { pertenencia } & \end{array}$ & $\begin{array}{l}\text { Acuden a rituales como rogativas, transmiten medicina } \\
\text { mapuche a través de la lactancia, y consideran a las } \\
\text { yerbas medicinales como innocuas para la salud. } \\
\text { Respeta medicina occidental, pero al llegar a su hogar } \\
\text { acude a Machi (autoridad religiosa y curativa de la } \\
\text { cultura mapuche). } \\
\text { [A los } 2 \text { meses de vida de mi guagua acudimos donde } \\
\text { el Machi, ya que él nos está ayudando mucho, le ha } \\
\text { hecho ya tres rogativas a él, falta uno. En este } \\
\text { momento está tomando su lawen que es remedio...]. } \\
\text { [Cuando mi guagua estaba tomando leche, chiquitito, } \\
\text { menos de } 1 \text { año, él no le da remedio a la guagua, } \\
\text { solamente me daba a mí, a través de mí, yo le } \\
\text { traspasaba el remedio a él]. }\end{array}$ \\
\hline & $\begin{array}{l}\text { Complementariedad } \\
\text { de la atención }\end{array}$ & $\begin{array}{l}\text { En primera instancia acude a la Medicina occidental } \\
\text { luego a la medicina mapuche. } \\
\text { [Lo diagnosticaron en el Consultorio y después lo llevé } \\
\text { a la medicina mapuche y le dieron unos medicamentos } \\
\text { que son súper fuertes y con eso se normalizó]. } \\
\text { [Primero acudo a la medicina del hospital, médico y } \\
\text { después voy a una consulta de hierbatería o a la machi]. } \\
\text { El Médico no permitió recibir hierbas durante la } \\
\text { hospitalización. } \\
\text { [En el hospital, no, un tiempo consulté yo, pero según } \\
\text { dijeron que ellos no permitían tomar remedios de } \\
\text { medicina de machi dentro del hospital...estando } \\
\text { hospitalizado no permiten]. } \\
\text { Desconoce la existencia del facilitador intercultural. } \\
\text { Refiere la necesidad de un hablante mapuche en forma } \\
\text { permanente durante la hospitalización. } \\
\text { [Entonces también sería bueno de que hubiera una } \\
\text { persona hablante, aunque sea } 1 \text { o } 2 \text {, pero que esté ahí } \\
\text { permanente...en cada sala]. }\end{array}$ \\
\hline & $\begin{array}{ll}\text { Calidad } & \mathrm{de} \\
\text { atención } & \end{array}$ & $\begin{array}{l}\text { Atención hospitalaria buena. } \\
\text { No tiene quejas. } \\
\text { Está agradecida del equipo. } \\
\text { Las enfermeras la han educado. } \\
\text { Buena experiencia con atención de enfermeras, siempre } \\
\text { presentes y le dan ánimo. } \\
\text { [Se me han facilitado muchas cosas, se me ha } \\
\text { enseñado mucho por lo que tiene mi guagua, también la } \\
\text { atención, yo evalúo buena]. }\end{array}$ \\
\hline & Discriminación & $\begin{array}{l}\text { Tiene miedo a que la "reten" si dice que acude a la } \\
\text { Machi. Se queda callada. } \\
\text { Percibe que ha cambiado, antes mapuches y no } \\
\text { mapuches "no podían juntarse". }\end{array}$ \\
\hline
\end{tabular}




\section{Discusión}

La vivencia de madres mapuches es permeada por un fuerte arraigo a la cultura de origen y se aprecia que se acude a la medicina occidental, pero no se observa en los textos la adherencia a este, lo que podría mostrar cierta desconfianza a la medicina occidental. Si bien en la vivencia emerge la complementariedad de ambas atenciones de salud, las participantes requieren de la atención de la medicina mapuche para satisfacer sus necesidades de salud. En la vivencia "calidad de la atención", aunque se aprecia absoluta conformidad con la atención, esta puede estar enmascarada con la posición que adoptan las participantes frente a la medicina occidental. Finalmente, la vivencia "discriminación" se expresa como el miedo a hacer valer su cultura, manteniendo una posición pasiva.

Respecto al sentido de pertenencia, las participantes reconocen acudir a rogativas y rituales propios de la cultura mapuche. Manifiestan respeto por la medicina occidental, acudiendo a ella en una primera oportunidad y tras ello recurren a la medicina mapuche, que en muchas ocasiones les genera mayor confianza, considerando a la vez a las hierbas medicinales naturales inocuas para la salud. Destaca que acuden a la medicina mapuche no solo para tratar sus problemas de salud personales, sino también para sus hijos, trasmitiendo la medicina mapuche a través de la lactancia. Estos resultados concuerdan con otro estudio chileno propuesto por Ochoa et al. ${ }^{(13)}$, que aborda las percepciones de los usuarios indígenas urbanos adscritos al sistema público de salud, el cual expone que los participantes acudían a los servicios de salud en primera instancia, aunque con menor confianza en los resultados de sanación y solo con el fin de descartar alguna enfermedad grave, y se manifiesta la existencia de mayor confianza en el efecto de las hierbas, al ser percibidas como menos invasivas para el organismo. Los usuarios indígenas, si bien reconocen la labor de la medicina occidental, presentan un sentido de desconfianza hacia esta ${ }^{(14-15)}$ y por otra parte consideran que la práctica de la medicina mapuche actúa en su beneficio de manera integral, ya que vinculan el bienestar físico y espiritual de las personas, y su práctica en contextos urbanos permitirían la mantención de la identidad étnica, así como un núcleo de transmisión cultural ${ }^{(13)}$. En concordancia un estudio colombiano expone como logros de la medicina tradicional indígena la alta asistencia de usuarios, la confianza de los pacientes, la fácil accesibilidad y asequibilidad, la calidad humana, la disminución de costos y el control satisfactorio de enfermedades ${ }^{(16)}$.

En complementariedad de la atención, las participantes expresan que la primera consulta la realizan con medicina occidental, para luego recurrir a la medicina mapuche de forma complementaria, sin embargo, durante la hospitalización esta no se expresa, debido a la presión social ejercida por las normativas institucionales, agrava la situación el desconocimiento de la existencia de facilitadores interculturales, por lo que no se logra visualizar complementariedad entre ambos sistemas de salud en la atención brindada a los pacientes durante su hospitalización, a pesar de las políticas implementadas para potenciar la interculturalidad en los centros de salud (2). Asimismo, en la formación de profesionales de salud en Chile, solo una Universidad tiene incorporado el tema de Salud Intercultural en su Plan de Estudio y solo para la Carrera de Enfermería. Otras entidades educativas superiores contemplan este enfoque en los objetivos de sus programas, aunque su desarrollo como concepto y como práctica es insuficiente ${ }^{(17)}$. Situación preocupante debido a que el desarrollo de un enfoque de salud intercultural supone, entre otras condiciones, la formación y desarrollo de equipos de salud capaces de respetar, comprender y responder apropiadamente a las propuestas y necesidades de las comunidades y organizaciones indígenas ligadas al área de la salud ${ }^{(18-19)}$. Al respecto Ochoa et al. ${ }^{(13)}$, expone que actualmente se 
reconoce la coexistencia y el diálogo entre ambas medicinas, pero coexiste la incapacidad de parte del equipo de salud y funcionarios de incorporar las demandas del paciente indígena dentro el proceso y los espacios de atención pública o de comprender y legitimar la diversidad cultural, los conocimientos, creencias y prácticas propias del mundo indígena en Chile.

Otro aspecto de la vivencia, es la calidad de la atención, la que se ha calificado de carácter positivo, de forma que las participantes expresan su agradecimiento y valoran la labor del equipo de salud en la atención de sus hijos, así como las intervenciones educativas brindadas por las enfermeras, con las que establecieron una relación de apoyo. Considerando que la interculturalidad en salud constituye una herramienta para mejorar el acceso y la calidad de atención ${ }^{(14)}$. Chile ha impulsado diversas estrategias, para la incorporación del enfoque intercultural en los programas de salud, planteándose el mejoramiento de la calidad de atención, a través del trato al usuario, adecuación horaria y pertinencia cultural en la atención de salud a través del componente de equidad $^{(1)}$, pero a pesar de estas políticas en este estudio se observa que el modelo de salud pública muestra carencias para atender a las actuales demandas y realidad de la población indígena en centros hospitalarios en la región de la Araucanía, y llama la atención que este hecho no se exprese en la vivencia de calidad de atención, aspecto que, puede estar enmascarada con la posición que adoptan las participantes frente a la medicina occidental. Por otra parte, las deficiencias en la calidad de la atención, sí han sido reportadas en otras investigaciones; en un estudio boliviano se concluye que las mujeres indígenas que rechazaron el parto institucional, fueron motivadas por la deficiente calidad de la atención de los centros asistenciales, debido a que fueron víctimas de expresiones despectivas y descalificadoras tanto de ellas como de sus prácticas nativas de salud (20).

En estrecha relación a lo expuesto anteriormente surge en la vivencia, la discriminación, aspecto que se evidencia con las manifestaciones de temor de expresar y reconocer que se recurre a la medicina mapuche, ya que se percibe algún tipo de retroalimentación negativa desde la medicina occidental que les genera miedo. El temor de las participantes de expresar el conocimiento y práctica de la medicina mapuche, indudablemente refleja una vez más la incapacidad del modelo de salud pública de incorporar las demandas del paciente indígena, tal como lo expresa Ochoa et al. ${ }^{(13)}$, quien además, manifiesta que la población indígena sufre experiencias negativas en la atención de salud, incluyendo malos tratos, prácticas de discriminación, escaso acceso a horas y tratamientos médicos, trabas burocráticas, deficiente relación usuario/profesional, entre otras. Realidad que también se expresa en estudios internacionales, en donde se manifiesta la existencia de maltrato y discriminación sobre la población indígena ejercida por el propio personal sanitario, quienes deben asegurar el bienestar físico, psicológico y espiritual de los pacientes ${ }^{(16)}$. Como resultado de estas prácticas se generan temor y desconfianza hacia los trabajadores de salud, que con sus actos crean una barrera que dificulta que la población indígena busque en el sistema de salud la atención que necesita ${ }^{(21)}$.

De acuerdo a lo expresado se hace necesario que los equipos de salud, logren mayor comprensión de la diversidad, dinámica cultural y social en los que están insertos los usuarios indígenas, más aún aquellos profesionales de la salud que se desempeñan en zonas de alta concentración de población nativa, sectores en los que se requiere contar con equipos capaces de respetar, comprender y responder a las propuestas y necesidades de las comunidades $\mathrm{y}$ organizaciones indígenas, para lograr brindar un cuidado culturalmente congruente ${ }^{(21-22)}$. Lejos de esta realidad, hoy se habla de "ceguera cultural", en profesionales de salud, presentándose un déficit de conocimientos culturales y la inconsciencia de ello, lo que impide desde la práctica 
enfermera brindar cuidados con "coherencia cultural" (23-24). No obstante, en las últimas décadas los enfermeros han aumentado su interés en considerar la cultura en la asistencia sanitaria, influenciadas también por las tendencias migratorias, por lo que hoy son más conscientes de la necesidad de adquirir competencia cultural, que les facilite el establecimiento de las relaciones terapéuticas a través del entendimiento y la comprensión de la cultura, fortaleciendo de esta manera la práctica asistencial ${ }^{(25-27)}$. Cabe destacar que la competencia cultural es una capacidad que se desarrolla gradualmente y tiene como finalidad brindar atención de salud segura y de calidad a usuarios de diferentes orígenes culturales, encontrándose como atributos definitorios la conciencia cultural, sensibilidad cultural, conocimiento cultural y la habilidad cultural ${ }^{(26-28)}$.

Desde el punto teórico, la Teoría de la Diversidad y Universalidad de los cuidados Culturales de Madeleine Leininger apoya los resultados desde las concepciones de cultura y valores de las madres (creencias de la salud, la enfermedad y su modelo de conducta). Se devela la congruencia de los cuidados culturalmente pertinentes (beneficiosos y eficaces) y se explica el saber transcultural en las decisiones y acciones de las madres. Finalmente, el estudio intenta poner en relieve el cuidado cultural como teoría holística, que considera la vida humana en su plenitud y los factores de la estructura social, la trayectoria cultural, el contexto del entorno y las expresiones lingüísticas ${ }^{(6)}$.

\section{Conclusiones}

Se aprecia en las madres una vivencia ambigua y uno de sus elementos clave son los patrones del desarrollo, que responden a una lógica de conocimiento cultural, espiritual y social. Asimismo, la complementación es un desafío que requiere de la comprensión del contacto intercultural en torno al dialogo y respeto a la diversidad. Otro elemento es el patrón intercultural, referenciado desde la cultura de los dos mundos.

Descriptivamente se aprecia cierta vaguedad en el sentido de pertenencia a la medicina mapuche y occidental, por mayor confianza, fácil asistencia, accesibilidad, asequibilidad, buena calidad humana y costos menores en la primera ${ }^{(16)}$.

Se aprecia asimismo contrariedades, decepciones y complicaciones en legitimar la diversidad cultural y social, que se traducen en impedimentos para el éxito de las estrategias de coherencia cultural en salud, ya que emergen trazos de discriminación. La integralidad del mundo indígena se manifiesta en las personas a través de la expresión de los valores propios de su cultura. De la misma forma se observa que el modelo de salud pública es anacrónico para atender las actuales demandas y realidad de la población indígena, ya que se perpetúa una relación con deficiente comunicación entre usuario mapuche-equipos de salud.

Sugerencias: Preocupa el déficit de capacitación de los equipos en Salud Intercultural, dado que, para el éxito de esta práctica se requiere de profesionales y técnicos capaces de respetar, comprender y responder apropiadamente a las propuestas y necesidades de población indígena. La incapacidad del equipo de salud, para incorporar las demandas del paciente indígena, incide en legitimar la diversidad cultural, competencia, conciencia cultural, sensibilidad cultural, conocimiento cultural y habilidad cultural para una atención de salud segura y de calidad. En este aspecto enfermería debe liderar y posesionarse en el enfoque del cuidado cultural para lograr mayor comprensión de la diversidad como fenómeno humano, donde el cuidado se debe expresar 
con apertura, simpatía y generosidad, elementos clave para la seguridad, confianza, aceptación y colaboración en salud.

\section{Referencias bibliográficas}

1. Cheuquepán S, Henríquez J, Bustos B. Orientaciones Técnicas Programa Especial de Salud y Pueblos Indígenas. Guía Metodológica para la Gestión del Programa. [Internet]. Chile: Ministerio de Salud; 2016 [acceso 17 de diciembre de 2018]. Disponible en:http://www.bibliotecaminsal.cl/wp/wp-content/uploads/2018/01/030.OT-y-Guia-Pueblosindigenas.pdf

2. Ministerio de Salud. Norma General Administrativa $N^{\circ} 16$, Interculturalidad en los Servicios de Salud. [Internet]. Chile: Ministerio de Salud; 2006 [acceso 17 de diciembre de 2018]. Disponible en:https://www.minsal.cl/sites/default/files/files/Norma\%2016\%20Interculturalidad.pdf

3. Gobierno de Chile. Estrategia nacional de salud para el cumplimiento de los Objetivos Sanitarios de la Década 2011-2020. [Internet]. Chile: Ministerio de Salud; 2010 [acceso 12 de diciembre de 2018]. Disponible en: http://www.minsal.cl/portal/url/item/c4034eddbc96ca6de0400101640159b8.pdf

4. Subsecretaría de Redes Asistenciales. Informe de Descripción de Programas Sociales. Programa Especial de Salud y Pueblos Indígenas. [Internet]. Chile: Ministerio de Salud; 2016 [acceso 12 de diciembre de 2018]. Disponible en: http://www.programassociales.cl/pdf/2017/PRG2017_3_59209_2.pdf

5. Subsecretaría de Redes Asistenciales. Programa Especial de Salud y Pueblos Indígenas. [Internet]. Chile: Ministerio de Salud; 2013 [acceso 12 de diciembre de 2018]. Disponible en: https://www.araucanianorte.cl/images/PDF-WORD/Resol.Ex-N-20-del-17.01.2013Programa-PESPI.pdf

6. Leininger MM. Teoría de la diversidad y de la universalidad de los cuidados culturales. En Raile M, Marriner A. Modelos y teorías en Enfermería. 7a ed. Barcelona: Elsevier; 2011. p. 454-479.

7. Boyle JS. Estilos de etnografía. En: Morse JM, compilador. Asuntos críticos en los métodos de investigación cualitativa. Medellín: Universidad de Antioquia; 2003. p.185-214.

8. Fariñas, G. Acerca del concepto vivencia en el enfoque histórico cultural. Revista Cubana de Psicología. 2005; 16 (3): 62-66.

9. Figueredo N. Investigación cualitativa en ciencias de la salud. Contribuciones desde la etnografía. Cuidados Humanizados [Internet]. 2017 [acceso 17 de junio de 2019]; 6(Núm. Especial): 14-19. doi: https://doi.org/10.22235/ech.v6iEspecial.1445

10. Knoblauch H. Etnografía enfocada. Forum Qualitative Sozialforschung / Forum: Qualitative Social Research [en línea]. 2005 [acceso 17 de junio de 2019]; 6(3). doi: http://dx.doi.org/10.17169/fqs-6.3.20

11. Hernández R, Fernández C, Baptista P. Metodología de la Investigación. 5a ed. México: McGraw-Hill; 2010. p. 471-478.

12. Lolas F, Quezada A. ¿Qué hace que la investigación clínica sea ética? Siete requisitos éticos. Pautas éticas de investigación en sujetos humanos: nuevas perspectivas. [Internet] 2003 [acceso 18 de diciembre de 2018]; 84-95. Disponible en: 
http://www.libros.uchile.cl/files/presses/1/monographs/258/submission/proof/files/assets/basi c-html/page $2 . h t m l$

13. Ochoa G, Inalef R, Valenzuela A. Percepción de salud - enfermedad de usuarios indígenas urbanos adscritos al sistema público de salud. [Internet]. Chile: Ministerio de Salud; 2012 [acceso 12 de diciembre de 2018]. Disponible en: https://germina.cl/wpcontent/uploads/2012/07/estudio_percepcion_salud_enfermedad.pdf

14. Patiño A, Sandín M. Diálogo y respeto: bases para la construcción de un sistema de salud intercultural para las comunidades indígenas de Puerto Nariño, Amazonas, Colombia. Salud colect. [en línea]. 2014 [acceso 14 de enero de 2019]; 10(3): 379-396. Disponible en: http://www.scielo.org.ar/scielo.php?script=sci_arttext\&pid=S1851-82652014000300008

15. Caceres P, Ribas A, Gaioli M, Quattrone F, Macchi A. The state of the integrative medicine in Latin America: The long road to include complementary, natural, and traditional practices in formal health systems. Eur J Integr Med [en línea]. 2015 [acceso 4 de diciembre de 2018]; 7(1):5-12. doi: https://doi.org/10.1016/j.eujim.2014.06.010

16. Cardona Jaiberth, Rivera Y, Carmona J. Expresión de la interculturalidad en salud en un pueblo emberá-chamí de Colombia. Rev. cub. salud pública [en línea]. 2015 [acceso 4 de diciembre de 2018]; 41(1):77-93. Disponible en: http://scielo.sld.cu/scielo.php?script=sci_arttext\&pid=S0864-34662015000100008

17. Painemilla A. Sanhueza A., Venegas J. Abordaje cualitativo sobre la incorporación del enfoque de salud intercultural en la malla curricular de universidades chilenas relacionadas con zonas indígenas. Rev Chil Salud Publica [en línea]. 2013 [acceso 4 de diciembre de 2018]; 17(3): 237-244. doi:10.5354/0719-5281.2013.28607

18. Fleckman J, Dal M, Ramirez S, Begalieva M, Johnson C. Intercultural competency in public health: a call for action to incorporate training into public health. Front Public Health [en línea]. 2015 [acceso 4 de diciembre de 2018]; 2 (210).doi: https://doi.org/10.3389/fpubh.2015.00210

19. Vargas S, Berúmen L, Arias I, Mejía Y, Realivázquez L, Portillo R. Determinantes sociales de la atención comunitaria: percepciones de la enfermera e indígenas Rarámuris. CULCyT [en línea]. 2015 [acceso 4 de diciembre de 2018]; 57(12). Disponible en: http://erevistas.uacj.mx/ojs/index.php/culcyt/article/viewFile/857/811

20. Aizenberg L. Hacia una aproximación crítica a la salud intercultural. Rev. latinoam. poblac. [en línea]. 2011 [acceso 4 de diciembre de 2018]; 9(5): 49-69. Disponible en: https://www.redalyc.org/articulo.oa?id=323827305003

21. Hasen F, Interculturalidad en Salud: Competencias en Prácticas de Salud con Población Indígena. Cien. enferm. [en línea]. 2012 [acceso 4 de diciembre de 2018]; 18(3): 17-24. Disponible en: https://scielo.conicyt.cl/scielo.php?script=sci_arttext\&pid=S071795532012000300003

22. Subsecretaría de Redes Asistenciales. Orientaciones para la Implementación del Modelo de Atención Integral de Salud Familiar y Comunitaria [en línea]. Chile: Ministerio de Salud; 2013 [acceso 12 de diciembre de 2018]. Disponible en: https://www.minsal.cl/portal/url/item/e7b24eef3e5cb5d1e0400101650128e9.pdf

23. Muñiz N. Cuidados Enfermeros y Coherencia Cultural. Ene [en línea]. 2014 [acceso 4 de diciembre de 2018]; 8(1). doi: http://dx.doi.org/10.4321/S1988-348X2014000100004

24. Duan Y. A concept analysis of cultural competence. Int J Nurs Sci [en línea]. 2016 [acceso 14 de enero de 2019]; 3(3): 268-273. doi: https://doi.org/10.1016/j.ijnss.2016.08.002 
25. Castrillón E. La enfermera transcultural y el desarrollo de la competencia cultural. Cul Cuid [en línea]. 2015 [acceso 14 de julio de 2019]; 19(42): 128-136. doi:10.14198/cuid.2015.42.11

26. Castillo J. El Cuidado Cultural de Enfermería: Necesidad y Relevancia. Rev haban cienc méd [en línea]. 2008 [acceso 4 de diciembre de 2018]; 7(3). Disponible en: http://scielo.sld.cu/scielo.php?script=sci_arttext\&pid=S1729-519X2008000300003

27. Romero MN. Investigación, Cuidados enfermeros y Diversidad cultural. Index Enferm [en línea]. 2009 [acceso 4 de diciembre de 2018]; 18(2): 100-105. Disponible en: http://scielo.isciii.es/scielo.php?script=sci_arttext\&pid=S1132-12962009000200007

28. Henderson S., Barker M., Mak A. Strategies used by nurses, academics and students to overcome intercultural communication challenges. Nurse Educ Pract [en línea]. 2016 [acceso 14 de enero de 2019]; 16(1): 71-78. doi: https://doi.org/10.1016/j.nepr.2015.08.010

Cómo citar: Rivas Riveros E, García Silva V, Catalán Melinao Y. Vivencias de madres mapuches durante la hospitalización de sus hijos, en un hospital de alta complejidad del sur de Chile. Enfermería: $\quad$ Cuidados Humanizados. 2020; 9(1): 33-43. Doi: https://doi.org/10.22235/ech.v9i1.2147

Contribución de los autores: a) Concepción y diseño del trabajo, b) Adquisición de datos, c) Análisis e interpretación de datos, d) Redacción del manuscrito, e) Revisión crítica del manuscrito. E.R.R. ha contribuido en a,b,c,d,e; VGS en a,b,c,d,e; YCM en a,b,c,d,e.

Editora científica responsable: Dra. Natalie Figueredo 